\title{
Imaging translocator protein expression with positron emission tomography
}

\author{
Catriona Wimberley ${ }^{1,2} \cdot$ Irene Buvat ${ }^{3} \cdot$ Hervé Boutin $^{4,5,6}$
}

Published online: 3 November 2021

(c) The Author(s), under exclusive licence to Springer-Verlag GmbH Germany, part of Springer Nature 2021

The $18 \mathrm{kDa}$ translocator protein (TSPO) is constitutively expressed at low levels in healthy brain tissue predominantly in endothelial cells and is over-expressed in activated glial cells [1]. TSPO has been demonstrated as a biomarker in neurological and neurodegenerative diseases that have a component of neuroinflammation. For this reason, TSPO has been extensively used as a target for PET imaging in preclinical and clinical research protocols exploring neurological and neurodegenerative diseases over the past 30 years. The first PET tracer used to image TSPO was $\left[{ }^{11} \mathrm{C}\right]-(\mathrm{R})-\mathrm{PK} 11195$. Over the last 15 years, an increased interest in neuroinflammation as contributor to a broad range of neurological conditions has led to a strong interest in TSPO imaging. A swath of research to replace $\left[{ }^{11} \mathrm{C}\right]-(\mathrm{R})-\mathrm{PK} 11195$ with new tracers with lower non-specific binding, and hence with higher signal-to-noise ratio, has led to the emergence of more than 40 PET tracers with their own benefits and drawbacks. One consistent challenge for most of the new tracers developed is a sensitivity to the human

This article is part of the Topical Collection on Neurology

$\bowtie$ Hervé Boutin

herve.boutin@manchester.ac.uk

1 Edinburgh Imaging QMRI, BioQuarter, University of Edinburgh, Edinburgh EH16 4SB, UK

2 Centre for Clinical Brain Sciences, Chancellor's Building, BioQuarter, University of Edinburgh, Edinburgh EH14 4SB, UK

3 Institut Curie, Université PSL, Inserm, U1288 LITO, Orsay, France

4 Division of Neuroscience and Experimental Psychology, School of Biological Sciences, Faculty of Brain and Mental Health, University of Manchester, Manchester M13 9PL, UK

5 Wolfson Molecular Imaging Centre, University of Manchester, Manchester M20 3LJ, UK

6 Geoffrey Jefferson Brain Research Centre, Manchester Academic Health Science Centre, Northern Care Alliance \& University of Manchester, Manchester, UK genetic polymorphism rs6971 which leads to two different affinity states, with subjects having TSPO with high affinity, low affinity, or a mixture of the two. This can lead to the exclusion of research participants expressing the TSPO low affinity binding site, which has been shown to be present in about $10 \%$ of the population [2]. Despite the strong push in development of new tracers targeting TSPO expression, the function and behaviour of TSPO in normal or diseased states are still not fully understood. The interpretation of the TSPO PET signal depends on an understanding of the biological and pathological processes involved in TSPO expression and over-expression. The cellular source of TSPO is highly dependent on species $[1,3,4]$ and on the pathology or animal model. Furthermore, there are discrepancies in the field about the most appropriate PET quantification methods for different TSPO radiotracers due to the complexity of the target in healthy and pathological states.

In this series of reviews and articles, we aimed to include articles that would give the state-of-the-art regarding different aspects of TSPO PET imaging, address associated challenges, and present the most recent advances in the field. Amongst the included articles is a review explaining the cellular source of TSPO expression in health and diseases by Nutma et al. [1] Another review by Chauveau et al. [5] discusses how the $\left[{ }^{11} \mathrm{C}\right]-(\mathrm{R})-\mathrm{PK} 11195$ challengers developed over the past 15 years [6] have been implemented —or notin clinical studies and whether they live up to the promise of providing an improved signal-to-noise ratio when compared to $\left[{ }^{11} \mathrm{C}\right]-(\mathrm{R})-\mathrm{PK} 11195$. The challenge of quantification and kinetic modelling of TSPO PET is addressed in a review by Wimberley et al. [7] where the different methods and models used for quantification of TSPO PET in the literature are described. The advantages and limitations of the methods in the current literature are outlined to aid researchers to choose methods that will allow them to generate the most relevant parameters and pharmacological/biological indices for their studies. A further article on the topic of quantification outlines the requirements for implementation of the 
supervised cluster analysis method [8] for the identification of an appropriate pseudo-reference region [9]. We have also included reviews of the use of TSPO PET imaging in several neurological diseases (apart from Alzheimer's disease, for which there are a number of reviews already $[10,11])$ such as glioma [12], epilepsy [13], psychiatric conditions [14], and substance abuse [15]. One final review, by Van Camp et al. [16], describes the use of TSPO PET as a tool to study neuroinflammation in preclinical models where in vivo imaging enables longitudinal studies.

Beyond the reviews, the original articles included in the series cover a range of topics including changes in the tracer distribution in schizophrenia, leading to a different interpretation of the kinetic modelling output and therefore of TSPO expression and potential role of neuroinflammation in schizophrenia [17]. One preclinical study describes the use of TSPO PET as well as supra-paramagnetic iron oxide MRI to look at both activated microglia and infiltrating macrophages for a preclinical model of multiple sclerosis [18] where the combination of the two biomarkers brings a stronger interpretation of the neuro-immune response in the model of disease. Two articles describe preclinical characterisation of novel TSPO tracers, both with low sensitivity to the human genetic polymorphism rs6971 [19, 20]. As mentioned before, the genetic polymorphism implies that a percentage of the population is generally excluded from research studies, and tracers that are not affected by the polymorphism are greatly needed.

Finally, we have included several articles that are nonbrain TSPO studies as there has been a recent surge to use TSPO radiotracers for imaging of pathologies in organs besides the brain, which poses different problems compared to brain imaging, such as radiotracer metabolites entering the tissue. This series includes original contributions at a preclinical level, with validation of a simplified quantification method for novel TSPO tracer, $\left[{ }^{18} \mathrm{~F}\right]-\mathrm{LW} 223$, in rat heart and brain for future whole body PET studies [21] as well as clinical studies for head and neck cancer with $\left[{ }^{18} \mathrm{~F}\right] \mathrm{F}-\mathrm{DPA}-$ 714 [22], and after liver transplant with [ $\left.{ }^{18} \mathrm{~F}\right] \mathrm{GE}-180$ [23].

Neuroinflammation has gathered an increasing interest as potential contributor to almost every neurological disease from acute conditions such as stroke to chronic degenerative diseases such as Alzheimer's or Parkinson's disease and has been under-investigated in some conditions such as frontotemporal dementia (FTD) or motor neurone disease (MND). Because neuroinflammation can be driven by peripheral inflammation, the interplay between peripheral and central immune responses has also attracted a growing interest in research addressing neurological conditions. Whole body TSPO PET might play a strong role in future work investigating the inflammatory network in all kinds of pathology from neurological disorders to immune system disorders and even cancer and heart disease. As described in a number of articles in the series, the interpretation of the PET signal is not trivial for TSPO PET and attention should be paid to the cellular source of TSPO expression, the quantification, the species and pathology under study, and, in humans, the genetic polymorphism leading to binding affinity sensitivity.

For all these reasons, we believe that this series will be of great interest to scientists working in the field of neuroinflammation. The potential of this target as a biomarker of neuroinflammation and whole body immune response, as well as the time and money invested in developing radiotracers, indicates that TSPO PET imaging is set to continue to be a key component of the arsenal of useful methods for studying neuroinflammation.

\section{Declarations}

Ethics approval Institutional Review Board approval was not required because the paper is an Editorial.

Consent to participate Not applicable.

Conflict of interest The authors declare no competing interests.

\section{References}

1. Nutma E, Ceyzeriat K, Amor S, Tsartsalis S, Millet P, Owen DR, et al. Cellular sources of TSPO expression in healthy and diseased brain. Eur J Nucl Med Mol Imaging. 2021. https://doi.org/ 10.1007/s00259-020-05166-2.

2. Owen DR, Yeo AJ, Gunn RN, Song K, Wadsworth G, Lewis A, et al. An 18-kDa translocator protein (TSPO) polymorphism explains differences in binding affinity of the PET radioligand PBR28. J Cereb Blood Flow Metab. 2012;32(1):1-5. https://doi. org/10.1038/jcbfm.2011.147.

3. Nutma E, Gebro E, Marzin MC, van der Valk P, Matthews PM, Owen DR, et al. Activated microglia do not increase $18 \mathrm{kDa}$ translocator protein (TSPO) expression in the multiple sclerosis brain. Glia. 2021;69(10):2447-58. https://doi.org/10.1002/glia.24052.

4. Owen DR, Narayan N, Wells L, Healy L, Smyth E, Rabiner EA, et al. Pro-inflammatory activation of primary microglia and macrophages increases $18 \mathrm{kDa}$ translocator protein expression in rodents but not humans. J Cereb Blood Flow Metab. 2017;37(8):2679-90. https://doi.org/10.1177/0271678X17 710182 .

5. Chauveau F, Becker G, Boutin H. Have (R)-[(11)C]PK11195 challengers fulfilled the promise? A scoping review of clinical TSPO PET studies. Eur J Nucl Med Mol Imaging. 2021. https://doi.org/ 10.1007/s00259-021-05425-w.

6. Chauveau F, Boutin H, Van Camp N, Dolle F, Tavitian B. Nuclear imaging of neuroinflammation: a comprehensive review of [11C]PK11195 challengers. Eur J Nucl Med Mol Imaging. 2008;35(12):2304-19. https://doi.org/10.1007/ s00259-008-0908-9.

7. Wimberley C, Lavisse S, Hillmer A, Hinz R, Turkheimer F, Zanotti-Fregonara P. Kinetic modeling and parameter estimation of TSPO PET imaging in the human brain. Eur J Nucl Med Mol Imaging. 2021. https://doi.org/10.1007/s00259-021-05248-9. 
8. Turkheimer FE, Edison P, Pavese N, Roncaroli F, Anderson AN, Hammers A, et al. Reference and target region modeling of [11C](R)-PK11195 brain studies. J Nucl Med. 2007;48(1):158-67.

9. Schubert J, Tonietto M, Turkheimer F, Zanotti-Fregonara P, Veronese M. Supervised clustering for TSPO PET imaging. Eur J Nucl Med Mol Imaging. 2021. https://doi.org/10.1007/ s00259-021-05309-z.

10. Janssen B, Mach RH. Development of brain PET imaging agents: strategies for imaging neuroinflammation in Alzheimer's disease. Prog Mol Biol Transl Sci. 2019;165:371-99. https://doi.org/10. 1016/bs.pmbts.2019.04.005.

11. Boche D, Gerhard A, Rodriguez-Vieitez E, Faculty M. Prospects and challenges of imaging neuroinflammation beyond TSPO in Alzheimer's disease. Eur J Nucl Med Mol Imaging. 2019;46(13):2831-47. https://doi.org/10.1007/ s00259-019-04462-w.

12. Zinnhardt B, Roncaroli F, Foray C, Agushi E, Osrah B, Hugon $\mathrm{G}$, et al. Imaging of the glioma microenvironment by TSPO PET. Eur J Nucl Med Mol Imaging. 2021. https://doi.org/10.1007/ s00259-021-05276-5.

13. Bouilleret V, Dedeurwaerdere S. What value can TSPO PET bring for epilepsy treatment? Eur J Nucl Med Mol Imaging. 2021. https://doi.org/10.1007/s00259-021-05449-2.

14. De Picker LJ, Haarman BCM. Applicability, potential and limitations of TSPO PET imaging as a clinical immunopsychiatry biomarker. Eur J Nucl Med Mol Imaging. 2021. https://doi.org/ 10.1007/s00259-021-05308-0.

15. Leroy C, Saba W. Contribution of TSPO imaging in the understanding of the state of gliosis in substance use disorders. Eur J Nucl Med Mol Imaging. 2021. https://doi.org/10.1007/ s00259-021-05408-x.

16. Van Camp N, Lavisse S, Roost P, Gubinelli F, Hillmer A, Boutin H. TSPO imaging in animal models of brain diseases. Eur J Nucl Med Mol Imaging. 2021. https://doi.org/10.1007/ s00259-021-05379-z.

17. Marques TR, Veronese M, Owen DR, Rabiner EA, Searle GE, Howes OD. Specific and non-specific binding of a tracer for the translocator-specific protein in schizophrenia: an [11C]-PBR28 blocking study. Eur J Nucl Med Mol Imaging. 2021. https://doi. org/10.1007/s00259-021-05327-x.
18. Coda AR, Anzilotti S, Boscia F, Greco A, Panico M, Gargiulo $\mathrm{S}$, et al. In vivo imaging of CNS microglial activation/macrophage infiltration with combined [(18)F]DPA-714-PET and SPIO-MRI in a mouse model of relapsing remitting experimental autoimmune encephalomyelitis. Eur J Nucl Med Mol Imaging. 2021;48(1):40-52. https://doi.org/10.1007/s00259-020-04842-7.

19. Ramakrishnan NK, Hird M, Thompson S, Williamson DJ, Qiao L, Owen DR, et al. Preclinical evaluation of (S)-[(18)F]GE387, a novel $18-\mathrm{kDa}$ translocator protein (TSPO) PET radioligand with low binding sensitivity to human polymorphism rs6971. Eur J Nucl Med Mol Imaging. 2021. https://doi.org/10.1007/ s00259-021-05495-w.

20. Lee SHDN, Laquintana V, Mangiatordi GF, Lopedota A, Lopalco A, Cutrinelli A, Franco M, Delre P, Song IH, Kim HW, Kim SB, Park HS, Kim K, Lee S-Y, Youn H, Lee BC, Kim SE Radiosynthesis and characterization of [18F]BS224: a next-generation TSPO PET ligand insensitive to the rs6971 polymorphism. Eur J Nucl Med Mol Imaging. 2021.

21. MacAskill MG, Wimberley C, Morgan TEF, Alcaide-Corral CJ, Newby DE, Lucatelli C, et al. Modelling [(18)F]LW223 PET data using simplified imaging protocols for quantification of TSPO expression in the rat heart and brain. Eur J Nucl Med Mol Imaging. 2021. https://doi.org/10.1007/s00259-021-05482-1.

22. Tuominen S, Keller T, Petruk N, Lopez-Picon F, Eichin D, Loyttyniemi E, et al. Evaluation of [(18)F]F-DPA as a target for TSPO in head and neck cancer under normal conditions and after radiotherapy. Eur J Nucl Med Mol Imaging. 2021;48(5):1312-26. https://doi.org/10.1007/s00259-020-05115-z.

23. Dirks M, Buchert R, Wirries AK, Pflugrad H, Grosse GM, Petrusch C, et al. Reduced microglia activity in patients with long-term immunosuppressive therapy after liver transplantation. Eur J Nucl Med Mol Imaging. 2021. https://doi.org/10.1007/ s00259-021-05398-w.

Publisher's note Springer Nature remains neutral with regard to jurisdictional claims in published maps and institutional affiliations. 